\title{
The Management of Complicated Celiac Disease
}

\author{
A. Al-toma W.H.M. Verbeek C.J.J. Mulder \\ Department of Gastroenterology, VU University Medical Center, Amsterdam, The Netherlands
}

\begin{abstract}
Key Words
Autologous stem cell transplantation - Gluten-free diet $\cdot$ Celiac disease $\cdot$ Non-responsive celiac disease . Refractory celiac disease, pathogenesis $\cdot$ Cladribine . Enteropathy-associated T-cell lymphoma
\end{abstract}

\begin{abstract}
Refractory celiac disease (RCD) is being defined as persisting or recurring villous atrophy with crypt hyperplasia and increased intraepithelial lymphocytes (IELs) in spite of a strict gluten-free diet (GFD) for $>12$ months or when severe persisting symptoms necessitate intervention independent of the duration of the GFD. RCD may not respond primarily or secondarily to GFD. All other causes of malabsorption must be excluded and additional features supporting the diagnosis of CD must be looked for, including the presence of antibodies in the untreated state and the presence of celiac-related HLA-DQ markers. In contrast to patients with a high percentage of aberrant T-cells, patients with RCD I seem to profit from an immunosuppressive treatment. RCD II is usually resistant to medical therapies. Response to corticosteroid treatment does not exclude underlying enteropathy-associated T-cell lymphoma. Cladribine seems to have a role, although it is less than optimal in the treatment of these patients. It may be considered, however, as the only treatment thus far studied that showed significant reduction of aberrant $T$ cells, seems to be well tolerated, and may have beneficial long-term effects in a subgroup of patients showing significant reduction of the aberrant T-cell population. Au-
\end{abstract}

tologous stem cell transplantation (ASCT) seems promising in those patients with persisting high percentages of aberrant $T$ cells. The first group of patients treated with ASCT showed improvement in the small intestinal histology, together with an impressive clinical improvement. However, it remains to be proven if this therapy delays or prevents lymphoma development.

Copyright $\odot 2007$ S. Karger AG, Basel

\section{Introduction}

Celiac disease (CD) is lifelong inflammatory condition of the gastrointestinal tract that affects the small intestine in genetically susceptible individuals. On small bowel biopsy there is a characteristic, although not specific, mucosal lesion that impairs nutrient absorption by the involved bowel. Prompt improvement of nutrient absorption and healing of the characteristic intestinal mucosal lesion is seen upon withdrawal of dietary gluten [1].

Non-responsive celiac disease (NCD) can be described in terms of the clinical scenario of a lack of initial response to a prescribed gluten-free diet (GFD), or the recurrence of symptoms despite maintenance of GFD in a patient who responded initially to GFD [2]. Although clinical improvement is usually followed by histological improvement most of the time, on occasions there is evidence for histological improvement with persistence of clinical symptoms that could be related to other causes 
$[3,4]$. Clinical improvement is usually evident within the first few weeks of starting GFD; however, it might take up to 2 years before a complete restoration of intestinal mucosa is evident [5].

Specific definition of refractory celiac disease (RCD) is missing in the literature. We define true RCD as persisting or recurring villous atrophy with crypt hyperplasia and increased intraepithelial lymphocytes (IELs) in spite of a strict GFD for $>12$ months or when severe persisting symptoms necessitate intervention independent of the duration of the GFD [6]. RCD may not respond primarily or secondarily to GFD [7]. All other causes of malabsorption must be excluded and additional features supporting the diagnosis of CD must be looked for, including the presence of antibodies in the untreated state and the presence of celiac-related HLA-DQ markers.

Currently, two categories of RCD are being recognized: type I without aberrant T cells and type II with aberrant $T$ cells detected by immunophenotyping by flow cytometric analysis or immunohistology of the intestinal mucosa [5]. Arbitrarily, based on our own experience, a percentage of aberrant cells CD7+CD3- of CD103+ IEL or cytoplasmic CD3+ surface CD3- \% of CD103+ IEL of $\leq 10 \%$ has been regarded as normal, and $>20 \%$ as definitively abnormal.

\section{Pathogenesis of RCD}

\section{Genetic and Environmental Factors}

The environmental factor is mainly ingestion of gluten, while several genes contribute to the genetic predisposition [8]. The main genetic factors, as mentioned before, are given HLA-DQ genes, i.e. the genes encoding DQ2 or DQ8 in the HLA complex on 6p21. Approximately $95 \%$ of celiacs have a DQ2 comprised of $\mathrm{DQB1}{ }^{*} 02$ and DQA $1^{*} 05$. A small number of individuals lacking either of those heterodimers have DQB1*302 or DQA1*03 alone $[9,10]$. Gene dosage also affects CD susceptibility, heterodimer comprised of DQB1 ${ }^{*} 02$ and DQA $1^{*} 05$ and most of the remaining $5 \%$ have a DQ8 heterodimer. Homozygous individuals who carry DQB1*02 and DQA $1^{*} 05$ in cis on both chromosomes have a greater risk of developing complicated forms of CD [11]. Non-HLA complex genes seem to contribute, but the nature and effects of these genes are less well known. The identification and knowledge of the function of additional genetic factors should improve the understanding of the actual pathogenesis of $\mathrm{CD}$ and lead to new diagnostic strategies in case finding and screening high-risk groups.

The Management of Complicated Celiac Disease

\section{Diagnostic Approach to RCD}

\section{Revision of the Initial Celiac Disease}

In a patient with villous atrophy refractory to a GFD, the first step requires to reassess the initial diagnosis of $\mathrm{CD}$ in order to exclude other diseases, such as giardiasis, tropical sprue, postinfectious diarrhea, collagenous sprue, protein intolerance, tuberculosis (including atypical), AIDS, common variable immunodeficiency syndrome, Whipple's disease, radiation enteritis, immunoproliferative small intestinal disease, Crohn's disease, eosinophilic gastroenteritis and autoimmune enteropathy.

The presence of circulating antigliadin, antiendomysium (EMA) or anti-tissue transglutaminase (tTG) antibodies before the onset of the GFD, an HLA-DQ2 or HLA-DQ8 status and an initial clinical and histological improvement after a strict GFD are strongly suggestive of $\mathrm{CD}$. Regarding the histological features, an increased number of IELs ( $>30$ lymphocytes per 100 epithelial cells) are seen in almost all active CD patients [1].

\section{Assessment of the Gluten-Free Diet}

The most important cause of non-responsive celiac patient is failure to adhere to a GFD, which has been reported in up to $50 \%$ of adult CD patients [12]. The presence of persisting circulating EMA or tTG antibodies is strongly suggestive of dietary mistakes [12]. However, the absence of circulating antibodies cannot rule out minor, inadvertent or voluntary, ingestion of gluten in the diet. On the other hand, persisting antibody titers may also be found in rare patients on a strict GFD with RCD and especially EMA [3]. tTG antibodies mostly return to normal within 2-3 months. A careful dietary inquiry performed by a skilled dietician in CD should be performed as the first line of investigation in a supposed refractory CD patient.

\section{Exclude Other Causes of Diarrhea with/without Villous Atrophy}

In case of persisting diarrhea despite demonstrable improvement in the histologic lesion and exclusion of dietary mistakes, other associated disorders should be considered. Well-known causes responsible for symptoms mainly include microscopic colitis and more rarely intermittent pancreatic insufficiency in $\mathrm{CD}$, secondary lactase deficiency, bacterial overgrowth, coexisting inflammatory bowel disease, irritable bowel syndrome but also anal incontinence $[4,13]$.

Dig Dis 2007;25:230-236 
There are many other causes of villous atrophy besides CD [6]. Clinical history should investigate longer stays near the equator for detection of tropical sprue. Small bowel enteropathy seems to occur often in southern parts of Africa. Giardiasis should be excluded by immunofluorescence of stool samples and may be diagnosed by duodenal histology. Crohn's disease with involvement of the duodenum may mimic or even coexist with $\mathrm{CD}$. The term collagenous sprue should be used with caution, as this disease is not an established independent entity. A subepithelial matrix broader than $10-20 \mu \mathrm{m}$ should point to the diagnosis of collagenous sprue. Deposition of excess of extracellular matrix underneath the basement membrane is an unspecific reaction, which can be seen in gluten-responsive CD, as well as in several other entities of RCD and also in enteropathy-associated T-cell lymphoma (EATL). Collagenous band-like structures regress to a large part in responsive $\mathrm{CD}$.

Autoimmune enteropathy is seen mainly in children and young adults, but may occur also in elderly patients. The histological picture often shows a diminished number of Paneth cells. The number of IELs often is normal and patients present frequently with concurrent autoimmune diseases.

We have to realize that villous atrophy has also been reported in association with the presence of a thymoma, with protein intolerance, in conjunction with common variable immunodeficiency syndromes and eosinophilic enteritis. In common variable immunodeficiency, antibody testing for $\mathrm{CD}$-associated antibodies is not useful. Only histological and clinical improvement on a strict GFD may reveal underlying CD in single cases of common variable immunodeficiency.

\section{Exclude Malignant Complications of Celiac Disease}

Unexplained weight loss, abdominal pain, fever and night sweating should alarm physicians of an overt EATL. Other markers for overt EATL may be positive stool blood tests, increased $\mathrm{LDH}$ or $\beta_{2}$-microglobulin $[14,15]$. In patients on GFD, EATL need not necessarily be accompanied by duodenal villous atrophy [16].

A high index of suspicion for an overt lymphoma should lead to an extensive work-up including upper and lower endoscopy, ENT workup, CT scan of thorax and abdomen with enteroclysis, videocapsule enteroscopy (VCE) and double balloon enteroscopy (DBE) in order to obtain histological specimens. In some cases, laparotomy, intraoperative enteroscopy and full-thickness biopsies are necessary, as the operative procedure may come to an earlier diagnosis which may be essential.
New advances in small bowel imaging including CT scan and MR enteroclysis can improve the diagnostic accuracy in these patients [17]. PET scan has been investigated in patients with EATL and RCD. Hadithi et al. [18], in a prospective cohort of 8 EATL patients and 30 patients with RCD, demonstrate that PET can visualize in all patients sites affected by EATL as confirmed on biopsy.

The diagnosis of overt T-cell lymphoma is based on histological and immunohistochemical features with mainly evidence of large or medium-size T-cell proliferation expressing a $\mathrm{CD} 3+\mathrm{CD} 8$ - and $\mathrm{CD} 103+$ phenotype. The majority presents as $\mathrm{CD} 3+, \mathrm{CD} 8-, \mathrm{CD} 30+$ large cell lymphoma, however small cell lymphomas often are CD3+, CD8+, CD30- $[14,15]$.

Diagnosis of small bowel adenocarcinoma may even be more difficult than lymphoma. Especially tumors located in the jejunum and ileum, which are not reached by standard endoscopic techniques, require extensive investigations. Diagnosis may often be made only after operative procedures. Obscure gastrointestinal bleeding, obstructive symptoms, stenotic lesions on radiological examinations and VCE retention should raise the suspicion of these malignancies (author's own experience).

\section{Evolving Role of Double Balloon Enteroscopy}

First described by Yamamoto et al. [19] in 2001, DBE is a new endoscopic technique with the potential to allow complete visualization of the entire small bowel. In the European retrospective study, enteroscopy was diagnostic in all patients suspected of having RCD [20].

\section{Establishing the Diagnosis of Refractory Celiac}

\section{Disease}

Finally, RCD is a diagnosis of exclusion, defined as a persisting villous atrophy that does not respond to a strict GFD. Demonstration of an aberrant clonal intraepithelial T-cell population and/or loss of antigen on IELs seem to characterize this patient population on high risk for development of overt lymphoma and differentiates RCD II from RCD I, which shows low or almost absent aberrant T cells. RCD II is also referred to as cryptic intestinal T-cell lymphoma; sprue-like intestinal T-cell lymphoma. Detection of a clonal T-cell population by testing for Tcell receptor (TCR) rearrangement is thought to be highly predictive of EATL development. However, oligo- or monoclonal IEL populations can be detected in the large majority of both RCD I and RCD II patients, also in patients that do not develop an EATL. Clonality is therefore of limited use in establishing the diagnosis of RCD and to predict the development of EATL. 


\section{RCD I vs. RCD |I}

\section{Clinical and Biological Behavior}

Patients with RCD I may represent an earlier stage of the disease than RCD II and the prognosis may be better, and the risk of developing an overt lymphoma is almost non-existent. In RCD I, adherence to the GFD should be carefully investigated since a strict GFD may induce remission in some patients.

In RCD I, patients often develop concomitant autoimmune diseases, infectious and thromboembolic complications. Retrospective data from our patient population suggest that RCD I patients have a mortality rate, which is not different from that of the general population (author's own experience). The presence of mucosal ulcerations (ulcerative jejunitis) should alert the doctor for the possible presence of an early EATL [21].

RCD II is mostly observed in adults and the mean age at diagnosis of RCD II is between 50 and 60 years, but younger cases may be observed. Most of the patients develop severe malabsorption with weight loss, abdominal pain and diarrhea. Some patients may also have skin lesions mimicking pyoderma gangrenosum or ulcerations mostly on the legs, arms and face, chronic chest or sinusoidal infections or unexplained fever. The link between CD and RCD II is usually suggested by the detection of circulating antigliadin, anti-EMA or anti-TG antibodies before the initiation of the GFD in almost two-thirds of patients, an HLA-DQ2 or HLA-DQ8 status in almost all patients [11] and an initial response on GFD in about onethird of patients with RCD II.

\section{Endoscopic and Radiological Features}

Usually in RCD I and II, the same pattern of villous atrophy is observed as in classical active $\mathrm{CD}$. The finding of mucosal ulcerations, mostly in the jejunum, defines the clinical picture of ulcerative jejunitis [21]. In some cases of RCD II also stomach and/or colonic ulcerations may be found [22]. Enteroscopy (push enteroscopy, DBE or VCE) should be performed in such patients with RCD II in order to search for overt lymphoma and ulcerative jejunitis CT scan with MRI enteroclysis may be useful to exclude overt lymphoma and may demonstrate a mesenteric cavitation syndrome and hyposplenism (volume $<100 \mathrm{~cm}^{3}$ ) in $30 \%$ of cases (author's own experience). Enlarged mesenteric lymph nodes often accompany RCD, without necessarily being specific for a T-cell lymphoma. Staging investigations as recommended for non-Hodgkin lymphomas should be performed including bone marrow aspiration, CT scan of the thorax and abdomen, sonography of the neck as well as ENT workup especially in RCD II patients.

\section{HLA-DQ Typing}

Typing of HLA-DQA1* and DQB1* alleles can be performed on whole blood samples. In our immunology laboratory, this typing is being performed with a combined single-stranded conformation polymorphism/heteroduplex method by a semiautomated electrophoresis and gel staining method on the Phastsystem (Amersham-Pharmacia-Biotech, Uppsala, Sweden) [11].

We found a highly significant correlation between HLA-DQ2 homozygosity and the development of serious complications of CD, in particular RCD II and EATL [11]. The link between HLA-DQ2 homozygosity and development of RCD II and CD-associated lymphoma of intraepithelial origin thus suggests that the strength of the gluten-specific T-cell response in the lamina propria directly or indirectly influences the likelihood of RCD II and lymphoma development. It has been reported earlier by Vader et al. [23] that HLA-DQ2 homozygous antigenpresenting cells induce higher T-cell proliferation and cytokine secretion than HLA-DQ2/non-DQ2 heterozygous antigen-presenting cells. This may explain the strongly increased risk for disease development in HLA-DQ2 homozygous individuals. This would indicate that the adherence to a GFD is particularly important for CD patients who are HLA-DQ2 homozygous.

\section{Small Intestinal Biopsies}

Upper gastrointestinal endoscopy should be performed in all patients. At least 10 duodenal biopsies are to be taken for histological, immunohistochemical and flow cytometric examination. Four to six biopsies are fixed and preserved in 10\% formalin for histopathological and immunohistochemical evaluation. Three to four biopsies for TCR gene rearrangement studies are taken separately, preserved on Histocon and frozen at $-20^{\circ} \mathrm{C}$. For immunophenotypical evaluation, 3-4 biopsies are taken and preserved in RPMI medium.

\section{Immunophenotyping of IEL}

Lymphocytes and enterocytes are isolated from 3 to 4 small intestinal biopsies. Intraepithelial localization of lymphocytes is confirmed by surface expression of CD103 ( $\alpha \mathrm{E} \beta 7$ integrin, a gut homing receptor for E-cadherin). Arbitrarily, a percentage of aberrant cells CD7+CD3- of CD103+ IEL or cytoplasmic CD3+ surface CD3- \% of $\mathrm{CD} 103+\mathrm{IEL}$ of $\leq 10 \%$ has been regarded as normal, and $>20 \%$ as definitely abnormal. 


\section{TCR Gene Rearrangement Study}

Clonality assessment for TCR- $\gamma$ gene rearrangements is done using the Biomed-2 multiplex TCR-PCR protocol. Detection of a clonal T-cell population by testing for TCR rearrangement is thought to be highly predictive of EATL development. However, oligo- or monoclonal IEL populations can be detected in the large majority of both RCD I and RCD II patients, also in patients that do not develop an EATL. Clonality is therefore of limited use in establishing the diagnosis of RCD and to predict the development of EATL $[24,25]$.

\section{RCD I vs. RCD II}

In RCD I, histology of the small bowel mucosa in most cases is indistinguishable from active untreated CD. The number of IELs may be lower than in RCD II and active $\mathrm{CD}$, although this has not been proven in prospective studies. The IEL phenotype is normal with the expression of surface CD 3 associated with surface CD 8 and TCR- $\beta$ as in classical active CD. The number of CD 8 - and TCR- $\beta$ positive IELs should exceed $50 \%$ of IELs and lower expression seems to be quite sensitive for differentiation from RCD II but not very specific.

In RCD II, an abnormal IEL clonal population may be observed in $80 \%$ of patients with RCD on small intestinal biopsies. Although these IELs have a normal cytological feature, they exhibit an abnormal IEL phenotype with the expression of intracytoplasmic CD3e, surface CD103 and the lack of classical surface T-cell markers such as CD8, CD 4 and TCR- $\alpha \beta$. Furthermore, the abnormal IEL phenotype is associated with clonal TCR gene rearrangement. This abnormal IEL population usually represents $>50 \%$ of the IELs and may also be observed in gastric and/or colonic epithelium in around two-thirds of patients and may be found in the PBL in one-third. It may be also detected in skin lesions or in the chest in single patients, suggesting that RCD II is a diffuse gastrointestinal disease.

The use of CD3 and CD8 on fixed biopsies is a very reliable method in order to assess the presence of this abnormal IEL phenotype, even retrospectively.

More recently, it has been shown on cell lines derived from clonal abnormal IEL that recurrent chromosomal abnormalities including a recurrent 1q trisomy may be found in these patients. The diagnostic yield of these cytogenetic features has not been evaluated so far. These chromosomal abnormalities, the clonality of the TCR gene and the loss of antigens on IELs, together with the frequent diffusion of the abnormal lymphocytes indicate, in spite of their normal cytology and low in situ prolif- erative capabilities, that this clonal IEL population can be considered as a cryptic intraepithelial lymphoma. This hypothesis is sustained by follow-up studies.

\section{Medical Treatment Options}

\section{Treatment of Refractory Celiac Disease I}

In contrast to patients with a high percentage of aberrant $\mathrm{T}$ cells, patients with RCD I seem to profit from an immunosuppressive treatment. According to the data of Goerres et al. [26], azathioprine should be first-line therapy after induction of clinical remission with corticosteroids. Dose and duration of treatment with azathioprine are not established.

In contrast to RCD II, long-term treatment with corticosteroids or locally acting budesonide may be considered only in patients who have contraindications to other immunosuppressants.

Cyclosporine A, infliximab and tacrolimus have been reported to be effective in case reports, but further data are required particularly in the light of severe side effects [27]. These agents should only be considered in case of clinical deterioration despite corticosteroid therapy or intolerance to azathioprine. Intestinal absorption of cyclosporine $\mathrm{A}$ is worse than that of tacrolimus, what has to be considered in the acute treatment. Parenteral administration of tacrolimus also has to be considered. Close monitoring of renal function is inevitable.

Treatment with infliximab may induce prompt clinical and histological response, but this effect has to be weighed against its possible acute allergic and chronic immunosuppressive side effects [28].

\section{Treatment of Refractory Celiac Disease II}

RCD II is usually resistant to medical therapies. Response to corticosteroid treatment does not exclude underlying EATL, which has been shown in single cases. In case of RCD II with persistent clinical symptoms and/or a high percentage of aberrant $\mathrm{T}$ cells in intestinal biopsies in spite of a corticosteroid treatment, more aggressive therapeutic schemes should be considered. However, no therapy seems to be curative in RCD II. Some patients may benefit from azathioprine [26]. Caution is needed in instituting immunosuppressive therapy, as this may induce a high risk of progression to an overt lymphoma.

In most cases, CHOP-like regimens have been applied, but also other agents used for nodal NHL may be applied. Maurino et al. [29] reported the results of treating 
7 RCD II patients with azathioprine. Clinical and histologic improvement was noted in 5 of 7 treated patients, although 3 patients died ( 1 from leukopenic fever and 2 died early). However, in their follow-up report on treating 13 patients with azathioprine, they reported a $46 \%$ mortality rate. A recent report on the antitumor necrosis factor agent infliximab for treatment of RCD has been published, but no data were provided on aberrant $\mathrm{T}$ cells ( $\mathrm{T}$ flow cytometry or immunohistology) [30]. Recognizing that some patients with RCD II, and especially with ulcerative jejunitis, are suffering from a low-grade EATL, we treated a group of these patients with cytotoxic chemotherapy. Cladribine (2-chlorodeoxyadenosine $(2-C D A))$ is a synthetic purine nucleoside with cytotoxic activity. 2-CDA is of proven value in the treatment of hairy cell leukemia. Pathologic cells in hairy cell leukemia are CD103 positive as in T cells in RCD II. In the past few years, clinical trials with 2-CDA have confirmed its effectiveness in selected autoimmune disorders. Seventeen patients received 2-CDA therapy [31]. This therapy was well tolerated without serious side effects. Six of 17 patients $(35.8 \%)$ responded with clinical improvement and another 6 had a significant decrease in aberrant Tcell percentages. Interestingly, 1 of our patients developed a complete clinical, immunologic (aberrant T-cell percentage decreased from 70 to $15 \%$ ), and histologic recovery (Marsh classification MIIIC-MI) and remained symptom-free during $>4$ years of follow-up evaluation. Furthermore, ulcerative jejunitis, an endoscopic feature of RCD II, was seen to disappear in the 5 patients (29.4\%) who had it initially and interestingly none of these 5 patients thus far developed EATL. Seven patients (41.1\%) developed EATL within 6-38 months after starting treatment and subsequently died despite multiagent chemotherapy (cyclophosphamide, adriamycin, vincristine, and prednisone). Although EATL was excluded adequately at inclusion, 3 patients died of EATL within 5-7 months after therapy. Whether 2-CDA has accelerated the development of lymphoma cannot be concluded confidently. Few cases of secondary malignancies after 2CDA through T-cell immunodepression have been reported. Thus, therapy with 2-CDA seems to have a role, although based on our data it is less than optimal in the treatment of RCD with aberrant $\mathrm{T}$ cells. It may be considered, however, as the only treatment thus far studied that showed significant reduction of aberrant $T$ cells seems to be well tolerated, and may have beneficial long-term effects in a subgroup of patients showing significant reduction of the aberrant T-cell population.

The Management of Complicated Celiac Disease
Autologous Stem Cell Transplantation in RCD II

In the 1990s, stem cell transplantation became an increasingly accepted treatment option for patients with severe autoimmune diseases refractory to conventional treatment. The application of this treatment option in gastroenterology has been explored in the last few years. We have tested the applicability of autologous stem cell transplantation (ASCT) in a selected group of refractory celiacs with aberrant $T$ cells [32].

Between March 2004 and March 2006, 7 patients were transplanted. EATL were excluded by endoscopic examination, computed tomography, body positron emission tomography and bone marrow biopsy. Stem cells were harvested from the peripheral blood after mobilization using G-CSF. The conditioning regimen consisted of Tcell depletion with fludarabine and myeloablation with melphalan. At follow-up, our patients showed improvement in the small intestinal histology, together with impressive clinical improvement as demonstrated by disappearance of diarrhea and abdominal pain, normalization of serum albumin, electrolytes and hemoglobin, increase in BMI and improvement of the performance status. Two years after transplantation, our first patient is showing further improvement in his immunopathology status as demonstrated in further decline in the percentage of aberrant $\mathrm{T}$ cells to $3 \%$ and histologically improved from Marsh III-A to Marsh I. We propose that enhanced apoptosis of activated but aberrant $\mathrm{T}$ cells has led to this late but remarkable decline. Our most recent patient with clinically short bowel syndrome is showing remarkable clinical, endoscopical and immunological improvement. Furthermore, the first 3 patients showed a significant increase in the percentage of CD8+ lymphocytes, which is seen as a marker of lymphocyte regeneration after ASCT. Absence of a demonstrable improvement in the surface expression of CD8 on the IEL might be regarded as a poor prognostic indicator of response; this is only to be proved or disproved on longer-term follow-up.

Although the short-term results in these patients are promising, follow-up at present is too short to permit firm conclusions as to efficacy. The selection of patients for this treatment should be restricted to those patients with a substantial population of aberrant $T$ cells, even after therapy with 2-CDA who have a greater tendency to progress to highly lethal EATL.

\section{Follow-Up and Overt Lymphoma in Refractory Celiac}

Disease

RCD II is a serious disorder with a 5-year survival less than $50 \%$ and the most frequent cause of death is the oc- 
currence of an overt T-cell lymphoma and recurrent infections. The presence of an abnormal clonal IEL population is significantly associated with a poor survival and a high risk of progression to overt lymphoma. The same clonal TCR gene rearrangement initially identified in patients with clonal RCD may be subsequently observed in lymphomatous specimens suggesting a continuum between RCD and high-grade lymphoma. The risk of devel- oping an overt T-cell lymphoma in patients with RCD II seems to be favored by immunosuppressive drugs. ASCT in RCD II patients seems to promising. Whether a close monitoring with videocapsule and/or PET scan are capable of detecting earlier lesions in RCD before development of an overt lymphoma and result in better outcome, remains to be answered.

\section{References}

1 Working Group of the United European Gastroenterology Week in Amsterdam: When is a coeliac a coeliac? Eur J Gastroenterol 2001;13:1123-1128.

-2 Schuppan D, Kelly CP, Krauss N: Monitoring non-responsive patients with celiac disease. Gastrointest Endosc Clin N Am 2006;16: 593-603.

3 Wahab PJ, Crusius JB, Meijer J, Wand Mul- 14 der CJJ: Gluten challenge in borderline gluten-sensitive enteropathy. Am J Gastroenterol 2001;96:1464-1469.

-4 Abdulkarim A, Burgart L, See J, Murray J: Etiology of non-responsive celiac disease: results of a systematic approach. Am J Gastroenterol 2002;97:2016-2021.

5 Cellier C, Delabesse E, Helmer C, et al: Refractory sprue, coeliac disease, and enteropathy-associated T-cell lymphoma. Lancet 2000;356:203-208.

-6 Daum S, Cellier C, Mulder CJ: Refractory coeliac disease. Best Pract Res Clin Gastroenterol 2005; 19:413-424.

7 Marsh MN: Gluten, major histocompatibility complex and the small intestine. A molecular and immunobiologic approach to the spectrum of gluten sensitivity ('celiac sprue'). Gastroenterology 1992;102:330-354.

-8 Van Belzen MJ, Meijer JW, Sandkuijl LA, et al: A major non-HLA locus in celiac disease maps to chromosome 19. Gastroenterology 2003;125:1032-1041.

-9 Mazzarella G, Maglio M, Paparo F, et al: An immunodominant DQ8-restricted gliadin peptide activates small intestinal immune response in in vitro cultured mucosa from HLA-DQ8 positive but not HLA-DQ8 negative coeliac patients. Gut 2003;52:57-62.

10 Karell K, Louka AS, Moodie SJ, et al: European genetics cluster on celiac disease. HLA types in celiac disease patients not carrying the DQA $1^{*} 05-\mathrm{DQB1}{ }^{*} 02(\mathrm{DQ} 2)$ heterodimer: results from the European Genetics Cluster on Celiac Disease. Hum Immunol 2003;64: 469-477.

- 11 Al-toma A, Goerres MS, Meijer JW, Pena AS, Crusius JB, Mulder CJ: Human leukocyte antigen-DQ2 homozygosity and the development of refractory celiac disease and enteropathy-associated T-cell lymphoma. Clin Gastroenterol Hepatol 2006;4:315-319.
12 Vahedi K, Mascart F, Mary JY, et al: Reliability of antitransglutaminase antibodies as predictors of gluten-free diet compliance in adult celiac disease. Am J Gastroenterol 2003;98:1079-1087.

13 Mulder CJ, Harkema IM, Meijer JW, De Boer NK: Microscopic colitis. Rom J Gastroenterol 2004;13:113-117.

14 Chott A, Dragosics B, Radaszkiewicz T: Peripheral T-cell lymphomas of the intestine. Am J Pathol 1992;141:1361-1371.

15 Daum S, Ullrich R, Heise W, et al: Intestinal non-Hodgkin's lymphoma: a multicenter prospective clinical study from the German Study Group on Intestinal Non-Hodgkin's Lymphoma. J Clin Oncol 2003;21:2740 2746.

16 Schmitt-Gräff A, Hummel M, Zemlin M, et al: Intestinal T-cell lymphoma: a reassessment of cytomorphological features in relation to patterns of small bowel remodelling. Virchows Arch 1996;429:27-36.

17 Tomei E, Diacinti D, Marini M, Mastropasqua M, Di Tola M, Sabbatella L, Picarelli A: Abdominal CT findings may suggest coeliac disease. Dig Liver Dis 2005;37:402406.

18 Hadithi M, Mallant M, Oudejans J, et al: ${ }^{18} \mathrm{~F}$ FDG-PET-fluorodeoxyglucose positron emission tomography versus computed tomography for the detection of enteropathyassociated T-cell lymphoma in refractory celiac disease. J Nucl Med 2006;47:1622-1627.

19 Yamamoto H, Sekine Y, Sato Y, et al: Total enteroscopy with a nonsurgical steerable double-balloon method. Gastrointest Endosc 2001:53:216-220.

20 Ell C, May A, Nachbar L, et al: Push-and-pull enteroscopy in the small bowel using the double-balloon technique: results of a prospective European multicenter study. Endoscopy 2005;37:613-616.

21 Ashton-Key M, Diss T, Pan L, et al: Molecular analysis of T-cell clonality in ulcerative jejunitis and enteropathy-associated T-cell lymphoma. Am J Pathol 1997;151:493-498.

22 Verkarre V, Asnafi V, Lecomte T, et al: Refractory coeliac disease is a diffuse gastrointestinal disease. Gut 2003;52:205-211.
23 Vader W, Stepniak D, Kooy Y, Mearin L, Thompson A, van Rood JJ, Spaenij L, Koning F: The HLA-DQ2 gene dose effect in celiac disease is directly related to the magnitude and breadth of gluten-specific T-cell responses. Proc Natl Acad Sci USA 2003;100: 12390-12395.

24 Bagdi E, Diss TC, Munson P, Isaacson PG: Mucosal intraepithelial lymphocytes in enteropathy-associated T-cell lymphoma, ulcerative jejunitis, and refractory celiac disease constitute a neoplastic population. Blood 1999;94:260-264.

25 Blumberg RS, Yockey CE, Gross GG, Ebert EC, Balk SP: Human intestinal intraepithelial lymphocytes are derived from a limited number of T-cell clones that utilize multiple V $\beta$ T-cell receptor genes. J Immunol 1993; 150:5144-5153.

26 Goerres MS, Meijer JW, Wahab PJ, et al: Azathioprine and prednisone combination therapy in refractory coeliac disease. Aliment Pharmacol Ther 2003;18:487-494.

27 Wahab P, Crusius J, Meijer J, et al: Cyclosporin in the treatment of adults with refractory coeliac disease - an open pilot study. Aliment Pharmacol Ther 2000;14:767-774.

28 Gillet HR, Arnott IDR, McIntyre M, et al: Successful infliximab treatment for steroidrefractory celiac disease: a case report. Gastroenterology 2002;122:800-805.

29 Maurino E, Niveloni S, Chernavsky A, et al: Azathioprine in refractory sprue: results from a prospective, open-label study. Am J Gastroenterol 2002;97:2595-2602.

- 30 Turner SM, Moorghen M, Probert CS: Refractory coeliac disease: remission with infliximab and immunomodulators. Eur J Gastroenterol Hepatol 2005;17:667-691.

- 31 Al-toma A, Goerres MS, Meijer JW, von Blomberg BM, Wahab PJ, Kerckhaert JA, Mulder CJ: Cladribine therapy in refractory celiac disease with aberrant $\mathrm{T}$ cells. Clin Gastroenterol Hepatol 2006;4:1322-1327.

32 Al-toma A, Visser O, van Roessel HM, von Blomberg BME, Scholten PET, Ossenkoppele GJ, Huijgens PC, Mulder CJJ: Autologous hematopoietic stem cell transplantation in four celiacs with aberrant $\mathrm{T}$ cells. Blood 2007;109:2243-2249. 